\title{
Variability in expiratory flow requirements among oscillatory positive expiratory pressure
}

\author{
Sherwin E. Morgan, RRT ${ }^{1}$, Steven Mosakowski, RRT, MBA ${ }^{1}$, Brenda L. Giles, MD², \\ Edward. Naureckas, $\mathrm{MD}^{3}$, Avery Tung, MD, FCCM ${ }^{4}$
}

\begin{abstract}
S.E. Morgan, S. Mosakowski, B.L. Giles, E. Naureckas, A. Tung. Variability in expiratory flow requirements among oscillatory positive expiratory pressure devices. Can J Respir Ther 2020;56:7-10. doi: 10.29390/cjrt-2019-025.

Introduction: Oscillatory positive expiratory pressure (OPEP) devices facilitate secretion clearance by generating positive end expiratory pressure. However, different device designs may produce different levels of expiratory pressure with the same expiratory flow rate. We bench tested four devices to determine the relationship between expiratory flow and expiratory pressure in each.

Methods: A bench model was created to test the gas flow rates required by different OPEP devices to generate target expiratory pressure. Four different devices were tested: Acapella ${ }^{\circledR}$ (DH Green, Smiths Medical), AerobiKa ${ }^{\circledR}$ (Monaghan Medical Corporation), VibraPEP ${ }^{\circledR}$ (Curaplex), and vPEPTM (D R Burton Healthcare). Each OPEP device was tested to determine the expiratory flow needed to generate expiratory pressure thresholds considered appropriate for OPEP therapy.

Results: The expiratory flow required to generate the same expiratory pressure thresholds varied considerably among devices. Valved OPEP devices such as the VibraPEP required less flow than mechanical devices such as the vPEP, Aerobika, and Acapella.

Discussion: In this bench test of OPEP devices, we found considerable variability in expiratory flow requirements needed to generate an expiratory pressure of $>10 \mathrm{~cm} \mathrm{H}_{2} \mathrm{O}$. Our finding suggests that smaller patients or those with limited expiratory airflow due to diseases such as COPD, obesity, chronic congestive heart failure, and restrictive lung disease may have better results when matched to OPEP devices requiring less expiratory airflow.
\end{abstract}

Key Words: oscillatory end expiratory pressure; expiratory airflow; pulmonary secretions

\section{INTRODUCTION}

Strategies to address pulmonary secretions in patients with lung disease have been recognized for more than 100 years [1]. Although initial techniques involved postural drainage and other passive approaches [1], informal technologies such as "blow bottles" to increase expiratory resistance and facilitate secretion clearance soon followed [2].

Commercially available positive expiratory pressure (PEP) devices also address secretion clearance by modifying expiratory resistance. These devices consist of a face mask or mouthpiece and a one-way valve to which expiratory resistors can be attached [3]. During PEP therapy the patient exhales against a fixed-orifice resistor, generating expiratory pressures ranging from 10 to $20 \mathrm{~cm} \mathrm{H}_{2} \mathrm{O}$ [3]. Oscillating positive expiratory pressure (OPEP) devices were introduced in Europe in the late 1980s [4] and Food and Drug Administration approved in the United States in 1994. OPEP devices periodically interrupt gas flow during active expiration to create pressure oscillations in addition to PEP. The vibration frequency ranges from 10 to $30 \mathrm{~Hz}$ with amplitudes ranging from 20 to 100 torr at flows of 10 and $25 \mathrm{~L} / \mathrm{min}[5,6]$.

Existing evidence is mixed regarding the effectiveness of OPEP in improving secretion clearance. In a 2009 study, Ramos et al. [7] reported that PEP levels as low as $15 \mathrm{cmH}_{2} \mathrm{O}$ effectively reduced sputum viscoelasticity after $10 \mathrm{~min}$. However, a 2017 Cochrane review observed that the quality of the evidence base supporting the efficacy of OPEP treatment was poor and that OPEP provided no clear benefit above other airway clearance techniques on health-related quality of life [7]. Nevertheless, OPEP therapy is clinically relevant in 2020 [8] and a 2015 review article recommended PEP/OPEP therapy to increase lung volume (functional residual capacity (FRC) and tidal volume), reduce hyperinflation, and improve airway clearance [9]. Suggested indications for airway clearance in that review included acute conditions such as copious secretions, ineffective cough, or an inability to mobilize secretions. Chronic indications for airway clearance therapy include cystic fibrosis, bronchiectasis, ciliary dyskinetic syndromes, and chronic obstructive pulmonary disease [9].

Practices vary with respect to the application of PEP therapy. However, Fagevik Olsén et al. described the procedure for PEP and or OPEP therapy as follows [9]:

(1) Start at resting expiration (FRC).

(2) Have the patient take in a breath that is larger than normal, but that does not completely fill the lungs.

(3) Perform a short breath hold.

(4) Exhale actively (3-4s) but not forcefully through the OPEP device, creating a PEP of $10-20 \mathrm{~cm} \mathrm{H}_{2} \mathrm{O}$ during exhalation. The length of inhalation should be approximately one-third of the total breathing cycle (inspiratory-to-expiratory ratio of $1: 3$ to $1: 4$ ).

(5) Perform 10-20 breaths.

${ }^{1}$ Department of Respiratory Therapy, 5841 S. Maryland Ave MC4028, University of Chicago, Chicago, IL 60637;

${ }^{2}$ Department of Pediatrics, 5841 S. Maryland Ave MC4028, University of Chicago, Chicago, IL 60637;

${ }^{3}$ Department of Medicine, 5841 S. Maryland Ave MC4028, University of Chicago, Chicago, IL 60637;

${ }^{4}$ Department of Anesthesia and Critical Care, 5841 S. Maryland Ave MC4028, University of Chicago, Chicago, IL 60637

Correspondence: Avery Tung, MD, FCCM, Department of Anesthesia and Critical Care, University of Chicago, 5841 S. Maryland Ave MC4028, Chicago,

IL 60637, USA. E-mail: atung@dacc.uchicago.edu

Published online at https://www.cjrt.ca on 04 March 2020 
The optimal volume of a breath during OPEP therapy has not been clearly identified in existing literature. In practice, the volume of breath needed to sustain a 3-4 s exhalation while maintaining $10-20 \mathrm{~cm} \mathrm{H}_{2} \mathrm{O}$ pressure will depend on the flow characteristics and design of the OPEP device. Commercially available OPEP devices can be largely categorized into two functional groups: mechanical and oscillating valve. Mechanical devices use either a fulcrum (Acapella ${ }^{\circledR}$, DH Green, Smiths Medical), rudder (AerobiKa ${ }^{\circledR}$, Monaghan Medical Corporation), or flapper (vPEP $^{\mathrm{TM}}, \mathrm{D}$ R Burton Healthcare) that occludes the airway and interrupts expiratory airflow. In contrast, an oscillating valve device (VibraPEP ${ }^{\circledR}$, Curaplex) relies on the passive reverberation of flow moving through an elastic valve to create PEP and oscillations. As a result, with use of an oscillating valve OPEP device a portion of the patient's flow (effort) is lost, and the ability of patients using the device to generate adequate pressures and flows may be compromised. This loss of kinetic energy is similar to the heat loss exhibited by electric motors due to inefficiency and would potentially lead to a need to maintain higher flow rates to generate the same positive expiratory pressure.

We hypothesized that as a result of the design differences described above different OPEP devices would require different flow rates to sustain an expiratory pressure $>10 \mathrm{cmH}_{2} \mathrm{O}$.

\section{Devices}

\section{METHODS}

OPEP devices from four differing manufacturers were tested (Acapella, AerobiKa, VibraPEP, and vPEP) (Figure 1). The AerobiKa, Acapella, and vPEP devices are mechanical devices whereas the VibraPEP operates via an oscillating valve mechanism. Five examples of each device from each of the manufacturers were tested.

\section{Equipment}

A bench model was created to determine the gas flow rates required to generate target expiratory pressures by different OPEP devices. The flow was generated using a Philips Respironics System One CPAP (Philips Respironics, Murrysville, PA) and was adjusted to maintain a target expiratory pressure. Flow monitoring was performed using a gas flow meter (TSI Inc, Shoreview, MN) and pressure gauge (CeComp, Libertyville, IL). A data acquisition system (NI USB 6001, National Instruments, Austin, TX) and LabVIEW Software (National Instruments) was used to record test values. A schematic of the configuration is shown in Figure 2 and a photograph of the actual experimental design is shown in Figure 3.

\section{Procedure}

Devices were connected to the distal end of the flow system, and the flow generator was set to achieve target pressures of $13 \mathrm{~cm} \mathrm{H}_{2} \mathrm{O}$ (low),

\section{FIGURE 1}

OPEP devices tested: a) Acapella ${ }^{\circledR}$ (DH Green, Smiths Medical), b) AerobiKa ${ }^{\circledR}$ (Monaghan Medical Corporation), c) VibraPEP ${ }^{\circledR}$ (Curaplex), and d) VPEPTM, D R Burton Healthcare

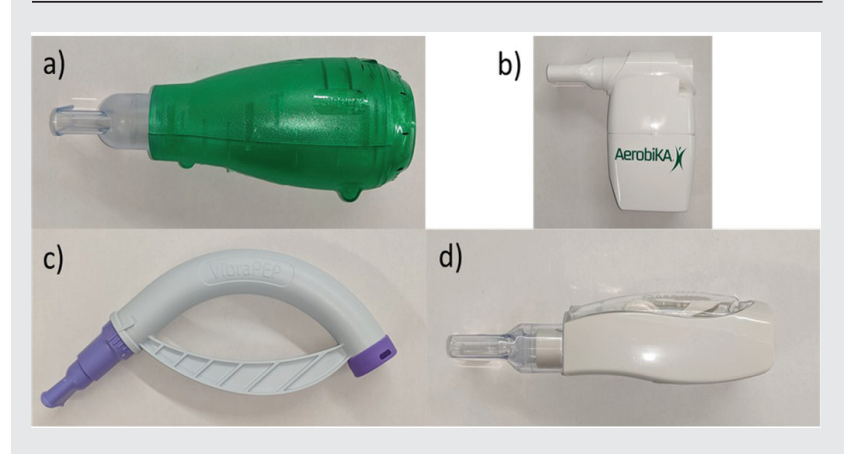

$15 \mathrm{~cm} \mathrm{H}_{2} \mathrm{O}$ (medium), and $17 \mathrm{~cm} \mathrm{H}_{2} \mathrm{O}$ (high). After steady-state pressures and flows were achieved, pressure and flow data were acquired electronically through the data acquisition device at a rate of $10 \mathrm{kHz}$ for a duration of $2 \mathrm{~s}$. This process was then repeated for each of the flow selector positions of the device. Each device was tested three times at each of the target pressures (except for the vPEP, which was not able to achieve the high target pressure with this test system).

\section{Data analysis}

Raw data files were analyzed to determine the steady-state pressure and flow using the arithmetic mean of the pressure and flow signals, and a Fourier Transform of the pressure signal was performed to determine the frequency of the oscillation of the device.

Data were then tabulated and analyzed with $\mathrm{R}$ version 3.6.0. Means are expressed as mean (95\% confidence interval). Analysis of variation (ANOVA) was used to compare group means at the 95\% confidence level. Pairwise t-test was used when ANOVA indicated differences between the groups and 0.05 taken as significant. The Bonferroni correction was used for multiple comparisons.

\section{Reproducibility}

\section{RESULTS}

To test the reproducibility of our measurements we performed three replicate measurements for each device measured at the medium pressure with the therapy selector set at position 1 as shown in Table 1. Replicate measurements were typically within $5 \%$ of the mean for each device.

\section{FIGURE 2}

Testing schematic for flow/pressure relationships among different OPEP devices. See text for device information.

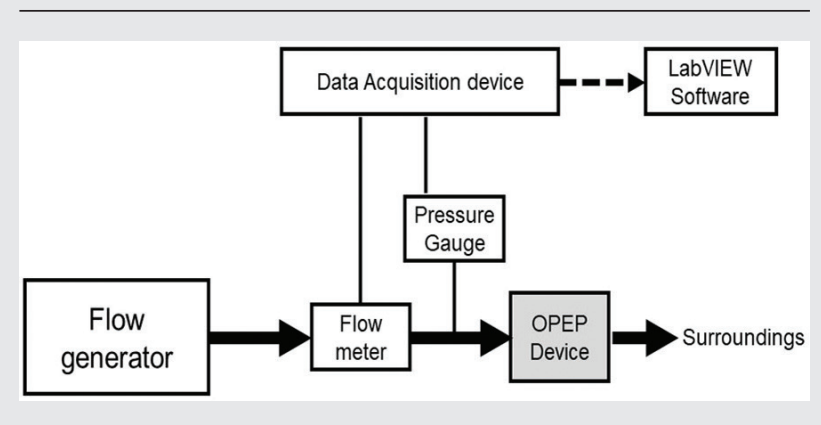

\section{FIGURE 3}

Testing setup including flow meter, pressure gauge and flow pathway. See text for device information.

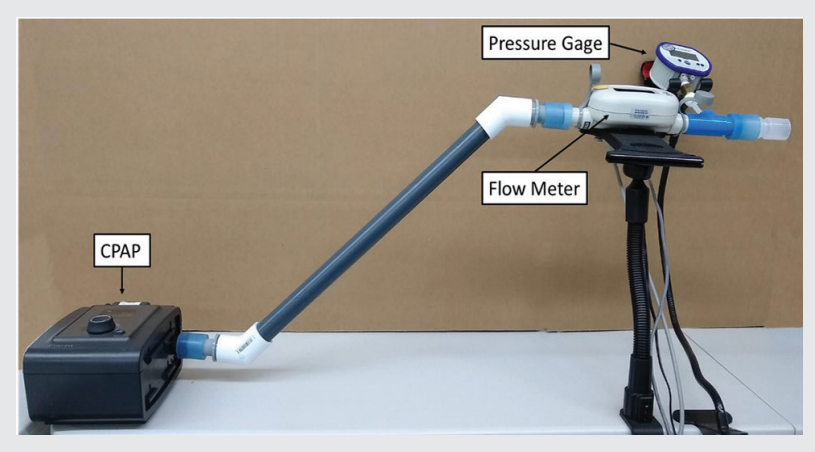




\section{TABLE 1}

Repeatability of measurements at the medium pressure with therapy selector set to position 1

\begin{tabular}{|c|c|c|c|c|}
\hline \multicolumn{5}{|c|}{ Steady-state flow (L/s) at medium pressure } \\
\hline & $\operatorname{Rep} 1$ & $\operatorname{Rep} 2$ & Rep 3 & Mean $(95 \% \mathrm{Cl})$ \\
\hline \multicolumn{5}{|l|}{ Acapella DH } \\
\hline Device 1 & 0.73 & 0.77 & 0.77 & $0.76(0.70-0.81)$ \\
\hline Device 2 & 0.81 & 0.79 & 0.80 & $0.80(0.77-0.83)$ \\
\hline Device 3 & 0.73 & 0.78 & 0.68 & $0.73(0.61-0.84)$ \\
\hline Device 4 & 0.81 & 0.78 & 0.77 & $0.79(0.73-0.84)$ \\
\hline Device 5 & 0.73 & 0.77 & 0.72 & $0.74(0.67-0.80)$ \\
\hline Overall mean & & & & $0.76(0.74-0.78)$ \\
\hline \multicolumn{5}{|l|}{ Aerobika } \\
\hline Device 1 & 0.91 & 0.93 & 0.94 & $0.93(0.88-0.97)$ \\
\hline Device 2 & 0.93 & 0.95 & 0.96 & $0.95(0.91-0.99)$ \\
\hline Device 3 & 0.96 & 0.95 & 0.94 & $0.95(0.93-0.96)$ \\
\hline Device 4 & 0.89 & 0.89 & 0.90 & $0.89(0.88-0.90)$ \\
\hline Device 5 & 0.93 & 0.95 & 0.96 & $0.95(0.91-0.99)$ \\
\hline Overall mean & & & & $0.93(0.92-0.95)$ \\
\hline \multicolumn{5}{|l|}{ VibraPEP } \\
\hline Device 1 & 0.43 & 0.40 & 0.45 & $0.43(0.37-0.49)$ \\
\hline Device 2 & 0.49 & 0.48 & 0.47 & $0.48(0.46-0.50)$ \\
\hline Device 3 & 0.47 & 0.48 & 0.48 & $0.48(0.46-0.49)$ \\
\hline Device 4 & 0.44 & 0.43 & 0.41 & $0.43(0.39-0.46)$ \\
\hline Device 5 & 0.46 & 0.49 & 0.50 & $0.48(0.43-0.53)$ \\
\hline Overall mean & & & & $0.46(0.44-0.48)$ \\
\hline \multicolumn{5}{|l|}{ vPEP } \\
\hline Device 1 & 0.97 & 0.99 & 0.98 & $0.98(0.95-1.01)$ \\
\hline Device 2 & 0.98 & 0.91 & 0.94 & $0.94(0.86-1.03)$ \\
\hline Device 3 & 0.94 & 0.95 & 1.00 & $0.96(0.88-1.04)$ \\
\hline Device 4 & 1.03 & 1.03 & 1.03 & $1.03(1.02-1.04)$ \\
\hline Device 5 & 0.89 & 0.88 & 0.88 & $0.88(0.87-0.90)$ \\
\hline Overall mean & & & & $0.96(0.93-0.99)$ \\
\hline
\end{tabular}

Note: Data are denoted as mean (95\% C.I.)

Steady-state flow rates

All devices were tested successfully, except vPEP at the high $\left(18 \mathrm{~cm} \mathrm{H}_{2} \mathrm{O}\right)$ target pressure. The resulting flow measurements are depicted in Figure 4, and the mean pressure, flow, and frequency measurements for therapy selector position 1 are shown in Table 2. Flow rates trend downward as the therapy selector position is increased, and the flow rates trend upward as the pressure is increased.

Flow rates needed to sustain the target pressure varied widely among devices (Table 2). The greatest difference was observed between the vPEP and the VibraPEP devices, with the vPEP requiring double the flow of

\section{FIGURE 4}

Steady state flow rates (liters/sec) for devices at the Low, Medium, and High target pressures. Error bars indicate 95\% C.I.

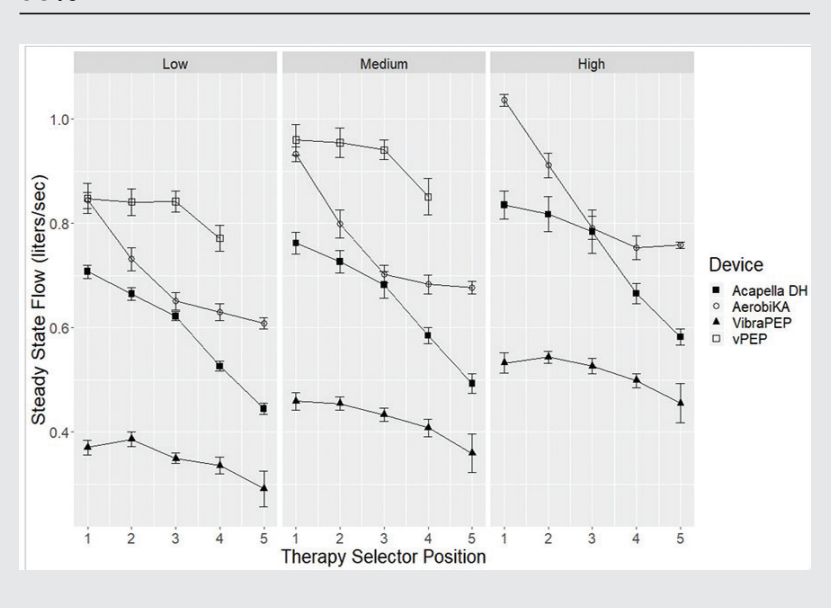

the VibraPEP. All comparisons using ANOVA within the low, medium, and high target pressure groups (by therapy selector position) were significantly different $(p<0.001)$ for all therapy selector positions. Pairwise comparisons found that flow requirements for the AerobiKA and vPEP devices were not significantly different when set to position 1 for both the low $(p=0.77)$ and medium $(p=0.051)$ pressure targets, and not different for the AerobiKA and Acapella DH devices when set to position 3 for the medium $(p=0.11)$ and high $(p=0.71)$ pressure targets. The VibraPEP device had the lowest steady-state flow rate and differed significantly from the other devices $(p<0.001$ for all pairwise comparisons to VibraPEP).

\section{DISCUSSION}

In our bench study of four commercially available OPEP devices, we found that expiratory flow requirements required to generate a target expiratory pressure of $>10 \mathrm{~cm} \mathrm{H}_{2} \mathrm{O}$ varied considerably among OPEP devices and therapy selector positions. As expected, steady-state flow rates required for expiratory pressure targets were higher for higher target pressures. In addition, increases in the therapy selector setting led to lower steady state flow rates. Valved OPEP devices such as the

TABLE 2

Performance characteristics of the OPEP devices for therapy selector position 1

\begin{tabular}{|c|c|c|c|}
\hline \multicolumn{4}{|c|}{ Performance characteristics at varying steady-state pressures, position 1} \\
\hline Low pressure & Steady-state pressure $\left(\mathrm{cm} \mathrm{H}_{2} \mathrm{O}\right)$ & Frequency $(\mathrm{Hz})$ & Steady-state flow (L/s) \\
\hline Acapella DH & $12.7(12.6-12.8)$ & $14.6(14.4-14.8)$ & $0.71(0.69-0.72)$ \\
\hline AerobiKA & $12.6(12.6-12.7)$ & $14.8(14.5-15.2)$ & $0.84(0.83-0.86)$ \\
\hline VibraPEP & $13.5(13.4-13.5)$ & $19.4(18.9-20.0)$ & $0.37(0.36-0.39)$ \\
\hline vPEP & $12.6(12.4-12.9)$ & $16.0(15.3-16.6)$ & $0.85(0.82-0.88)$ \\
\hline \multicolumn{4}{|c|}{ Medium pressure } \\
\hline Acapella DH & $14.5(14.4-14.6)$ & $16.0(15.8-16.3)$ & $0.76(0.74-0.78)$ \\
\hline AerobiKA & $14.4(14.3-14.5)$ & $16.0(15.6-16.4)$ & $0.93(0.92-0.95)$ \\
\hline VibraPEP & $15.3(15.3-15.3)$ & $19.3(18.7-20.0)$ & $0.46(0.44-0.48)$ \\
\hline vPEP & $14.6(14.3-15.0)$ & $16.9(16.1-17.6)$ & $0.96(0.93-0.99)$ \\
\hline \multicolumn{4}{|l|}{ High pressure } \\
\hline Acapella DH & $17.3(17.2-17.4)$ & $18.3(17.9-18.6)$ & $0.84(0.81-0.86)$ \\
\hline AerobiKA & $16.9(16.8-17.0)$ & $17.4(17.0-17.9)$ & $1.04(1.02-1.05)$ \\
\hline VibraPEP & $18.2(18.1-18.2)$ & $20.3(19.7-20.9)$ & $0.53(0.51-0.55)$ \\
\hline vPEP & N/A & N/A & N/A \\
\hline
\end{tabular}

Note: Data are denoted as mean (95\% C.I.). No data were collected for VPEP at the high target pressure. 
VibraPEP required less flow than mechanical devices such as the vPEP, Aerobika, and Acapella devices to generate an expiratory airway pressure of $>10 \mathrm{~cm} \mathrm{H}_{2} \mathrm{O}$.

This difference in flow requirements among devices can have clinical consequences. The flow volume required to generate 3-4 s of therapy may not be achievable by all patients depending on their weight and anticipated exhaled tidal volumes. Clinically, patients with lung disease are likely to experience an even larger disparity between the ability to generate flow and expiratory flow requirements as such patients are likely to have less ability to generate adequate expiratory flow than healthy patients.

Our data suggest that not all OPEP devices are suitable for all patients. Devices requiring a higher flow rate to generate therapeutic end-expiratory pressures such as the vPEP, Acapella, and Aerobika devices may be best suited to larger patients able to generate the expiratory flow rates and exhaled tidal volumes needed by those devices. For smaller patients, OPEP devices able to generate an appropriate airway pressure with lower expiratory flow rates may maximize the potential benefits of OPEP therapy. Elderly patients or those with obesity, chronic congestive heart failure, and restrictive lung and chest wall disorders may also not be able to generate the expiratory flow rates needed to sustain the target expiratory airway pressure suggested by Ramos et al. [7] and thus not reap the full benefit of OPEP.

In addition to not reaching target expiratory airway pressure, OPEP devices may alter respiratory homeostasis. A common adverse side effect identified in the warning sections of OPEP device instructions for users is increased work of breathing may lead to hypoventilation and hypercarbia. Because of the expiratory flow rates needed to maintain a therapeutic pressure in our study, patients may need to hyperventilate to generate the expiratory airflow needed to meet target pressure requirements. Our data suggest that such an adverse event would be less likely with OPEP devices that do not require a large airflow to generate the target pressure.

\section{CONCLUSION}

OPEP devices differ in the expiratory flow rate requirements needed to sustain a therapeutic end expiratory pressure. Lighter weight patients or those with lung diseases that limit expiratory flow may then be unable to generate target expiratory pressures with some OPEP devices. In our bench study, mechanical OPEP designs generally required higher flow rates than oscillating valve designs. When choosing an OPEP device an important variable is whether the patients can generate enough expiratory flow to meet end expiratory pressure targets.

\section{CONTRIBUTORS}

This manuscript represents original work and has not been published in whole or part elsewhere. Each author (SEM, SM, BLG, EN, AT) has contributed substantively to the design, analysis, and to drafting the work. All have approved the final version.

\section{COMPETING INTERESTS}

Dr. Tung receives a salary as executive editor for Critical Care for the journal Anesthesia $\mathbb{E}$ Analgesia. All authors have completed the ICMJE uniform disclosure form at www. icmje.org/coi_disclosure.pdf and declare: no financial relationships with any organizations that might have an interest in the submitted work in the previous 3 years and no other relationships or activities that could appear to have influenced the submitted work.

\section{FUNDING}

This study did not receive any specific grant from funding agencies in the public, commercial, or not-for-profit sectors.

\section{ETHICAL APPROVAL}

This study did not involve patient data or human subjects.

\section{REFERENCES}

1. Ewart W. The treatment of bronchiectasis and of chronic bronchial affections by posture and by respiratory exercises. Lancet 1901;2: 70-2. doi:10.1016/S0140-6736(01)85059-5

2. Colgran FJ, Mahoney PD, Fanning GL. Resistance breathing (blow bottle) and sustained hyperinflation in the treatment of atelectasis. Anesthesiology 1970;32:543-50. doi:10.1097/00000542-197006000-00017

3. AARC. Clinical practice guideline. Respir Care 1993;38(5):516-21.

4. Altaus P The bronchial hygiene assisted by the flutter VRP1 (Module regulator of a positive pressure oscillation on expiration). Eur Respir J 1989;2: (Suppl 8):693.

5. Volsko T, DiFiore J, Chatburn RL. Performance comparison of two oscillating positive expiratory pressure devices: Acapella versus flutter. Respir Care 2003;48(2):124-30.

6. Vines DL, Gardner DD. Egan's fundamentals of respiratory care. 11 edn., chapter 43, p. 981.

7. Ramos EM, Ramos D, Iyomasa DM, et al. Influence that oscillating positive expiratory pressure using predetermined expiratory pressures has on the viscosity and transportability of sputum in patients with bronchiectasis. J Bras Pneumol 2009;35(12):1190-7. doi:10.1590/S1806-37132009001200005

8. Bourbeau J, Mclvor RA, Devlin HM, Kaplan A. Oscillating positive expiratory pressure (OPEP) device therapy in Canadian respiratory disease management: Review, care gaps and suggestion for use. Can J Resp Crit Care Sleep Med 2019;3: 233-40. doi:10.1080/24745332.2018.1558426

9. Fagevik Olsén M, Lannefors L, Westerdahl E. Positive expiratory pressure - Common clinical applications and physiological effects. Respir Med 2015;109(3): 297-307. doi:10.1016/j.rmed.2014.11.003 